\title{
Network Pharmacology-Based Prediction and Verification of the Mechanism of Shikonin in the Treatment of Colorectal Cancer
}

\section{Zefeng Wang}

Shanghai University of TCM: Shanghai University of Traditional Chinese Medicine

Qianfei Cui

Shanghai University of TCM: Shanghai University of Traditional Chinese Medicine

Ling Shi

Honghe University

\section{Xiaojing Lu}

Shanghai University of TCM: Shanghai University of Traditional Chinese Medicine

\section{Yongjia Shi}

Shanghai University of TCM: Shanghai University of Traditional Chinese Medicine

\section{Wenjing Guo}

Gansu University of Traditional Chinese Medicine

\section{Peng Song ( $\nabla$ songp06@163.com )}

Gansu University of Traditional Chinese Medicine

\section{Research}

Keywords: Shikonin, Colorectal cancer, Network Pharmacology, IL6, AKT

Posted Date: July 23rd, 2021

DOl: https://doi.org/10.21203/rs.3.rs-711164/v1

License: (c) (i) This work is licensed under a Creative Commons Attribution 4.0 International License. Read Full License 


\section{Abstract}

Background: Zicao is the dried root of Lithospermum erythrorhizon Sieb, et Zucc, Arnebia euchroma (Royle) Johnst, or Arnebia guttata Bunge and is commonly used to treat viral infection, inflammation, arthritis, and cancer in traditional Chinese medicine. Shikonin (SKN), a naturally occurring naphthoquinone, is a major active chemical component isolated from Zicao and exhibits anticancer activity according to previous research. However, the underlying mechanism has not been elucidated, so further research is necessary to verify its traditional application. The purpose of the present study was to investigate the antitumor mechanism of SKN in colorectal cancer (CRC) through network pharmacology and experiments.

Methods: The SymMap database and GeneCards were adopted to predict the potential targets of SKN and CRC, while cotargets were obtained via a Venn diagram. The cotargets were imported from the String and DAVID websites, the protein-protein interaction (PPI) network was constructed, and Gene Ontology (GO) and Kyoto Encyclopedia of Genes and Genomes (KEGG) enrichment analyses were performed. With regard to the prediction of KEGG by DAVID, the compound-target-pathway network was generated by connecting potential pathways with the corresponding targets. According to the network pharmacological analysis, cytological experiments, real-time PCR (RT-PCR), and Western blot (WB) were used to verify the key signaling pathway.

Results: According to the network pharmacological analysis, the most relevant target of SKN to the treatment of colorectal cancer was IL6. GO and KEGG enrichment analyses indicated that various kinases and the PI3K/AKT signaling pathway were the most enriched molecules and pathways. SKN inhibited CRC cell (HT29 and HCT116) proliferation, migration, and invasion and promoted cell apoptosis by targeting IL6 and inhibiting the IL6R/PI3K/AKT signaling pathway.

Conclusions: SKN promotes apoptosis and suppresses CRC cell (HT29 and HCT116) activities through the PI3K-Akt signaling pathway. This research not only provides a theoretical and experimental basis for more in-depth studies but also offers an efficient method for the rational utilization of a series of traditional Chinese medicines as anti-CRC drugs.

\section{Highlight}

1. The antitumor mechanism and relevant target of shikonin in colorectal cancer (CRC) were predicted by network pharmacology.

2. The antitumor effect of shikonin was verified by cell experiments on CRC cells (HT29 and HCT116).

3. Pharmacological experiments demonstrated that shikonin inhibited CRC cell growth by targeting IL6 and inhibiting the IL6R/PI3K/AKT signaling pathway.

4. This research offers an efficient method for the rational utilization of a series of traditional Chinese medicines as anti-CRC drugs. 


\section{Introduction}

Colorectal cancer (CRC) is one of the most common intestinal malignancies worldwide. It has the third highest incidence among cancers and remains the second most common cause of cancer mortality [1]. The 5-year survival rate for patients with stage IV CRC is $<10 \%$, and there are no effective targeted drugs for alleviating advanced CRC in clinical treatment [2]. Therefore, it is essential to develop safer and more effective therapeutic targeted drugs to improve the survival rate of patients with advanced CRC.

Traditional Chinese medicine (TCM) has been used to effectively treat various diseases for more than 2500 years in China. It has been confirmed that TCM is very advantageous in the clinical application of cancer treatment and is widely accepted as a complementary and alternative therapy with obvious beneficial effects for cancer patients [3]. However, due to the complicated components of TCM, its underlying antitumor mechanisms are not completely understood. Currently, an increasing number of researchers have realized that network pharmacology can provide an effective approach to identify the association between targets, diseases, and drugs and reveal the mechanisms of TCM from a holistic view [4]. In addition, increasing evidence has shown that many drugs can achieve their therapeutic activities by regulating multiple targets. Therefore, network pharmacology is a feasible and comprehensive approach to investigate the antitumor mechanisms of TCM.

Lithospermiun erythrorhizon Sieb. et Zucc. (named Zicao in China) is the dry root of Arnebia euchroma (Royle) Johnst. or Arnebia guttata Bunge. Its dried roots are commonly used to "cool blood and invigorate blood, release toxins, and bring the rash to the surface" in TCM and have exact effects in the clinical applications of purpura, dermatitis, eczema, vaginitis, tumors, and acute and chronic hepatitis [5]. SKN is a natural naphthoquinone pigment compound purified from Lithospermum erythrorhizon. A modern study has indicated that SKN is a naturally extracted naphthoquinone pigment with many pharmacological functions, such as antibacterial, anti-inflammatory, antiviral, liver protection, antioxidation, antitumor and immune regulation [6]. It has already been reported that the anticancer effects of SKN against CRC cells are mediated by inducing cell apoptosis and inhibiting cell proliferation [7]. However, the mechanism and target of SKN antitumor activity still need to be further clarified.

The current study first studied the CRC targets of SKN through network pharmacology, then enriched the targets with bioinformatics, and preliminarily screened the IL6R/PI3K/AKT signaling pathway in apoptosis as the research object. In addition, the results were verified by pharmacology experiments [8].

\section{Materials And Methods}

\section{Prediction Targets of SKN and Components-Targets (C-T) network construction}

Identification of targets of effective molecules is a key step in understanding the mechanisms of action of compounds[9]. Here, the SymMap database (https://www.symmap.org/) integrates TCM with modern 
medicine through both internal molecular mechanisms and external symptom mapping and was used to search for targets. Currently, SymMap provides massive descriptive information on herbs, TCM symptoms, modern medicine symptoms, ingredients, targets, and diseases. It also provides pairwise relationships among all six types of components through direct association or indirect statistical inference. The 2D and 3D structures of SKN were obtained by PubChem, and the pictures were saved in an SDF format (Fig. 1A). Then, the 3D SDF format file was uploaded to the SymMap database, and the parameters were set according to the literature [3]. The targets of SKN were imported into Cytoscape 3.7.1 software to establish the C-T network.

\section{Prediction Targets of CRC}

GeneCards (https://www.genecards.org/) is a searchable, integrative database that provides comprehensive, user-friendly information on all annotated and predicted human genes. The predicted targets of CRC were collected from GeneCards. The targets of SKN were compared with CRC matching the common terms as potential targets [10].

\section{Acquisition of co-owner gene information for disease and active molecules}

The related targets of SKN were obtained by SymMap, and CRC-related genes were obtained by GeneCards. Next, it is necessary to determine the common pathogenic genes of disease and SKN. The coowner gene information was obtained by Bioinformatics \& Evolutionary Genomics, which is a website that can be used to calculate and draw Venn diagrams [11].

\section{PPI network}

The overlapping genes of SKN and CRC were considered hub genes and analyzed using online STRING to obtain the PPI, with the species limited to "Homo sapiens" and a confidence score $>0.990$. The TSV format file, which was downloaded from the STRING database, was imported into Cytoscape 3.7.1 for analysis. Cytoscape 3.7.1 is widely applied to network pharmacology research and used to construct and visualize the network. Furthermore, it provides a basic set of characteristics for data integration, analysis, and visualization for complicated network analysis. The key topological parameters (degree) characterize the most important nodes in the network; higher quantitative values of topological parameters indicate greater importance of the node [12].

\section{GO enrichment analysis}

GO analysis was performed using the online functional annotation and enrichment tool DAVID (https://david.ncifcrf.gov/). GO analysis consists of three different categories: GO terms including molecular function (MF), biological process (BP), and cellular components (CC) were identified $[13,14]$. GO terms with a $P$ value $<0.05$ were considered statistically significant [15].

\section{KEGG pathway enrichment analysis}


KEGG (http://www.genome.jp/kegg/) analysis was used to explore the biological pathways and potential biological functions on the basis of the enrichment analysis of functional items [16]. The data obtained from the KEGG database were used to perform pathway enrichment analysis using the DAVID system. KEGG pathways with enrichment $P$ values less than 0.05 were significant signaling pathways [17].

\section{Component-Target-Pathway (C-T-P) network construction}

The component-target-pathway network model was established using Cytoscape 3.7.1. In this network, nodes represent components $(C)$, targets $(T)$, and pathways $(P)$, and edges represent the interaction of $C$ T or T-P. Based on the results of KEGG enrichment analysis and the C-T database, the C-T-P interactions provide an overview of the mechanisms of SKM in the treatment of CRC.

\section{Molecular Docking}

Before docking, the 2D structures of SKN were transferred to 3D chemical structures, and energy minimization was performed for further docking. The crystal structure of IL6 (PDB ID: 409H) was extracted from PDB [18]. The protein structure was prepared for docking using the prepared protein tool implemented in Schrodinger Suites 2018-1. The binding site was defined as a sphere with a radius that stays within $2.42 \AA$ from the ligand using the Define and Edit Binding Site tool in Schrodinger Suites 2018-1. An OPLS3 force field was employed, and hydrogen atoms were added to the proteins. The binding site was defined as a sphere encompassing protein residues within $20 \AA$ of the original ligand. Default values were used for other parameters [19].

\section{Cell Cultures and SKN treatment}

Human CRC, HT29 and HCT116 cells were kindly provided by the Stem Cell Bank of the Chinese Academy of Sciences (Shanghai, China). They were cultured in McCoy's 5A medium (Sigma-Aldrich, St. Louis, MO, USA) supplemented with $2.2 \mathrm{~g} / \mathrm{L} \mathrm{NaHCO}_{3}, 10 \%$ FBS (Lot: 10270-106, Gibco, MD, USA), 2 mM glutamine, and 100 units/ml penicillin/streptomycin (Lot: 15140-122, Gibco, MD, USA) and maintained in an atmosphere of $5 \% \mathrm{CO}_{2}$ at $37^{\circ} \mathrm{C}$. The cells were detached by treatment with $0.25 \%$ trypsin/ $1 \mathrm{mM}$ EDTA (Lot: 25200-056, Gibco, MD, USA) and replated into different tissue culture plates for the following assays. Various SKNs were prepared by diluting $10 \mathrm{mM} \mathrm{SKN} \mathrm{(} \geq 98 \%$ purity, Lot: JOT-10104, Chengdu Pufei De Biotechnology Co. Ltd, Chengdu, China) with culture medium.

\section{Cytotoxicity assay and Ki67 staining}

Cytotoxicity was measured using a CCK-8 (Lot: MA0218-6, Meilun Biotech Co., Ltd, DaLian, China) assay as previously described [20]. HT29 and HCT116 cells were treated with the indicated concentrations of SKN for 24,48 , and $72 \mathrm{~h}$, and each assay was replicated 8 times. In addition, cell proliferation was assessed by Ki67 (Lot: 11882S, Cell Signaling Technology, U.S.A.) expression as previously described [20]. HCT116 cells were seeded in glass bottom dishes with polylysine (Lot: P4707, Sigma-Aldrich, MO, USA) and stained with fluorochrome-conjugated antibody for Ki67 after treatment with SKN for $48 \mathrm{~h}$. Finally, the cell nucleus was stained with DAPI (Lot: E607303-002, Proteintech Group, Inc., USA) for 30 min. Fluorescence signals were analyzed by using a confocal microscope (Nikon ECLIPSE T i2, Nikon 
Corporation, Tokyo, Japan), and Ki67 was quantified with ImageJ v5.0 software (National Institutes of Health).

\section{Cell cycle assay}

After incubation with the indicated concentrations of SKN for $48 \mathrm{~h}, \mathrm{HCT} 116$ cells were washed with cold phosphate-buffered saline (PBS) and fixed in 70\% ice-cold ethanol. All the samples were processed with a cell cycle kit (Lot: C1052, Beyotime, Shanghai, China) following the manufacturer's instructions, and the cells were filtered through a $30 \mu \mathrm{m}$ pore size nylon mesh before cell cycle analysis with a FACSCanto flow cytometer (CytoFLEX, Beckman, CA, USA).

\section{Cell apoptosis assay}

Cell apoptosis was measured using an Annexin V/propidium iodide (PI) staining kit (Lot: MA0220-2, Meilun Biotech Co., Ltd, DaLian, China) as previously described. The cells were harvested and washed with PBS after treatment with the indicated concentrations of SKN for $48 \mathrm{~h}$. Apoptotic cells were identified by double staining with fluorescein 5-isothiocyanate (FITC) (Lot: E00567-1632, eBioscience, CA, USA)conjugated annexin $\mathrm{V}$ and $\mathrm{PI}$ according to the manufacturer's instructions. Data were obtained and analyzed using a FACSCanto flow cytometer. In addition, cell apoptosis was assessed by Hoechst 33342 staining. The cells were treated with SKN for $48 \mathrm{~h}$, followed by the addition of $5 \mu \mathrm{g} / \mathrm{ml}$ Hoechst 33342 (Lot: C0003, Beyotime Biotechnology, Shanghai, China). After incubation for $15 \mathrm{~min}$, the cells were visualized and photographed under a fluorescence microscope (ECLIPSE Ti2, Nikon Corporation, Tokyo, Japan).

\section{Cell migration assay}

Cell migration was measured by wound healing assay as previously described. Monolayer HCT116 cells were wounded by scratching with pipette tips, and fresh McCoy's 5A medium containing different concentrations of SKN was added to the scratched monolayer cells. The migrated cells were imaged by microscopy (Nikon ECLIPSE Ti2, Nikon Corporation, Japan) after $24 \mathrm{~h}$. In addition, cell migration was assessed using a Transwell plate filter as described previously with some modifications. The bottom chambers were filled with McCoy's 5A medium containing 10\% FBS, and the top chambers were seeded with $100 \mu \mathrm{L}$ McCoy's 5A medium (without FBS) containing SKN and HCT116 cells. The migrated cells were fixed with $100 \%$ methanol and stained with $0.05 \%$ crystal violet (Lot: G1063, Solarbio Science \& Technology Co., Ltd. Beijing, China). The cells were quantified by manual counting and photographed under a microscope.

\section{Real-time quantitative PCR}

The mRNA expression of p53 was quantitatively determined at $12 \mathrm{~h}$ and $24 \mathrm{~h}$ after treatment with SKN. Total RNA in cells was extracted using an RNAiso Kit (cwbiotech, Jiang Su, China), and first-strand cDNA was synthesized with a PrimescripTM RT reagent kit (cwbiotech, Jiang Su, China). Real-time PCR was performed using a real-time PCR system (QuantStudio 6 Flex Real-time PCR System, Applied Biosystems, CA, USA). SYBR ${ }^{\circledR}$ Premix Ex TaqTM II (Takara Biotechnology) and specific primers were used in each 
PCR. The results after calibration with GAPDH expression were calculated using the $2^{-\triangle} \triangle \mathrm{Ct}$ method $\left(\triangle \Delta \mathrm{Ct}=\mathrm{Ct}_{\text {sample }}-\mathrm{Ct}_{\mathrm{GAPDH}}\right)$. The primer sequences for $\mathrm{p} 53$ were CCTCAGCATCTTATCCGAGTGG (forward) and TGGATGGTGGTACAGTCAGAGC (reverse), and the primer sequences for glyceraldehyde-3phosphate dehydrogenase (GAPDH) were AGAAGGCTGGGGCTCATTTG (forward) and AGGGGCCATCCACAGTCTTC (reverse) (Sangon Biotech, Shang Hai, China).

\section{Western blot quantification}

Protein expression was quantitatively determined by Western blotting at $48 \mathrm{~h}$ after SKN treatment. Briefly, the cells were lysed in ice-cold NP40 lysis buffer (Lot: P0013F, Beyotime Biotechnology, Shanghai, China) with proteinase inhibitor cocktail (Lot: P1045-1, Beyotime Biotechnology, Shanghai, China). Protein concentrations were measured with the Protein BCA Assay Kit (Lot: P0010S, Beyotime Biotechnology, Shanghai, China) and equalized before loading. Protein samples were boiled at $100^{\circ} \mathrm{C}$ for 5 min with loading buffer, subjected to $12 \%$ SDS-PAGE electrophoresis, electrotransferred onto a PVDF membrane, and incubated overnight at $4^{\circ} \mathrm{C}$ with primary antibodies. After washing with TBS, the membranes were incubated with horseradish peroxidase (HRP)-conjugated secondary antibodies (Lot: A0208 and A0216, goat anti-rabbit antibody and goat anti-mouse antibody, Beyotime Biotechnology, Shanghai, China, dilution $1: 5000$ ) for $2 \mathrm{~h}$ at $37^{\circ} \mathrm{C}$. The signal was detected using an enhanced chemiluminescence kit (Meilun Biotech Co., Ltd, DaLian, China) from an imaging workstation (Azure c600, Azure Biosystem ${ }^{\mathrm{TM}}, \mathrm{CA}$, USA).

\section{Results}

\section{SKN C-T Network}

A single component can also act on different targets, which makes it difficult to study the molecular mechanism of its pharmacodynamics. The emergence of network pharmacology research methods has brought convenience to pharmacological mechanism research of traditional Chinese medicine with multiple targets. To elucidate the molecular mechanism of SKN in CRC, we first established a C-T network by using the merge function in Cytoscape 3.7.1. The C-T network is shown in Fig. 1B and includes 30 nodes and 29 edges, providing evidence for SKN in the treatment of CRC.

\section{Acquisition of CRC targets}

GeneCards is an integrated database of human genes that includes disease relationships, and search keywords (colorectal cancer) are used to begin the search. In this study, the top 500 targets of CRC-related diseases were selected and used for intersection with drug targets.

\section{Acquisition of the co-owner target information for disease and SKN}

The overlapping targets of SKN and CRC were obtained by the website Bioinformatics \& Evolutionary Genomics, which can calculate the intersection(s) of a list of elements. Information on the 13 co-owner 
targets for disease and SKN is shown in Fig. 2.

\section{PPI network.}

The PPI network of cotarget genes was constructed using STRING, and the total interaction network contained 13 cotarget genes (node) and 122 interactions (edge) (Fig. 3A and B). According to the PPI data, the common target genes TP53, CCND1, CASP3, AKT1, IL4, CXCL8, IL6, IL10, KDR, PPARG, BCL2, PCNA, and EPHB2 might be potential target genes for SKN in the treatment of CRC [4].

\section{GO enrichment analysis}

GO enrichment analysis was performed to identify common features of 13 targets based on GO annotations. The number of $\mathrm{GO}$ terms that are significantly associated with the prescription targets for three ontologies (CC, BP, and MF) is 615, 602, and 458 [21]. The main BP terms included negative regulation of apoptotic process, response to drug, positive regulation of sequence-specific DNA binding transcription factor activity, response to glucocorticoid, and defense response to protozoan. The main CC terms included cytoplasm, nucleus, cytosol, extracellular region, and nucleoplasm. The main MF terms included protein binding, identical protein binding, enzyme binding, transcription factor binding, and ATP binding. These BPs, MFs and CCs were closely related to the treatment of CRC, and the results of GO enrichment analysis are shown in Fig. 4.

\section{KEGG enrichment analysis}

Through the DAVID database, 59 signaling pathways were obtained, the top 10 of which were chosen according to the $p$ value $(P$ value $<0.05)$, as shown in Fig. 5 . The top 10 significant signaling pathways included the PI3K-Akt signaling pathway, colorectal cancer signaling pathway, Jak-STAT signaling pathway, and some cancer-related signaling pathways [22]. These signaling pathways were closely related to the regulation of SKN in the treatment of CRC.

\section{C-T-P network}

Based on the above information, KEGG enrichment analysis revealed that 13 targets in the key subnetwork of PPIs were enriched in the top 10 signaling pathways of disease. Next, we constructed a compound-target-pathway network (Fig. 6) to provide a more comprehensive explanation of the mechanism of SKN in the treatment of CRC.

\section{Molecular docking verification}

The in silico study using molecular modeling was used to test the possible mode of action of SKN [23], and we searched the PDB database for the crystal structures of the most relevant potential targets of SKN in the treatment of CRC. The results of the molecular docking of SKN against the receptor IL6 (PDB: $409 \mathrm{H}$ ) showed that SKN could interact with IL6 receptors through hydrogen bonding, which was 
documented with 2 amino acid residues (LEU63 and GLU173). The molecular docking diagram of 3D and $2 \mathrm{D}$ is shown in Fig. 7. This result suggested that SKN can treat CRC by inhibiting interleukin 6 receptors, thereby inhibiting PI3K-AKT signaling pathways.

\section{Stable cytotoxicity of SKN toward CRC cells}

To determine the stable cytotoxicity of SKN, HT29 and HCT116 cells were treated with different concentrations of SKN for $24 \mathrm{~h}, 48 \mathrm{~h}$, and $72 \mathrm{~h}$ in triplicate in our study. The MTT results demonstrated concentration- and time-dependent cytotoxicity after exposure to SKN (Fig. 8A and B). The $\mathrm{IC}_{50}$ values for inhibiting CRC cells are shown in Fig. 8C. In addition, the cytotoxicity of SKN in HCT116 cells was higher than that in HT29 cells.

\section{SKN suppressed the proliferation of HCT116 Cells}

Ki67 is an essential nuclear antigen in tumor cells, which indicates the proliferation of tumor cells [24]. To investigate whether SKN suppressed the proliferation of HCT116 cells, we carried out an immunofluorescence staining assay to investigate the protein expression of Ki67. As shown in Fig. 9A and B, 2.5 and $5.0 \mu \mathrm{mol} / \mathrm{L}$ SKN significantly suppressed the protein expression of Ki67 in HCT116 cells.

To further detect the cell cycle arrest effect of SKN on HCT116 cells, flow cytometry analysis was employed to quantify cell cycle progression after treatment with SKN for $48 \mathrm{~h}$. These findings suggest cell cycle arrest at S phase. As shown in Fig. $9 \mathrm{C}$ and D, after treatment with $\mathrm{SKN}$, the percentage of the cell population at $\mathrm{S}$ phase was significantly increased and that at $\mathrm{G} 2$ phase was significantly decreased; however, cells at G1 phase had little change.

\section{SKN induced the apoptosis of HCT116 Cells}

To investigate whether SKN kills HCT116 cells predominantly through induction of apoptosis, Hoechst 33342 staining was employed. Untreated cells had round and homogenously stained nuclei, while cells treated with 2.5 and $5.0 \mu \mathrm{mol} / \mathrm{L}$ SKN showed marked DNA condensation and apoptotic bodies (Fig. 10A), which are essential characteristics of cell apoptosis.

To further confirm the induction of cell apoptosis by SKN, flow cytometry analysis was conducted after Annexin V-FITC/PI staining. We found that SKN triggered cell apoptosis in a dose-dependent manner (Fig. 10B and C). The cells treated with $5.0 \mu \mathrm{mol} / \mathrm{L} \mathrm{SKN}$ for $48 \mathrm{~h}$ showed over $50 \%$ apoptotic and necrotic cells. These findings suggest that cell apoptosis is an important mechanism of the antitumor activity of SKN.

\section{SKN inhibited the migration of HCT116 Cells}

The migration of tumor cells is an essential step in the complex process of tumor metastasis. Therefore, we carried out a wound healing assay to investigate the effects of SKN on cell migration and found that 2.5 and $5.0 \mu \mathrm{mol} / \mathrm{L}$ SKN obviously inhibited the migration of HCT116 cells (Fig. 11A and C). We also 
conducted Transwell assays to evaluate the ability of HCT116 cells to pass through the membrane barrier of the Transwell in the presence of various concentrations of SKN. The results showed that SKN significantly inhibited the migration properties of HCT116 cells in a dose-dependent manner (Fig. 11B and D).

\section{SKN promoted p53 expression by inhibiting the IL6R/PI3K/AKT pathway in HCT116 cells}

To investigate SKN's mechanisms underlying the proliferation, apoptosis, and migration of HCT116 cells, we tested whether SKN could modulate the levels of key signaling proteins. As shown in Fig. 12A, our results showed that protein expression levels of p53 and Caspase-3 increased. Moreover, decreases in MMP-9 and CDK2 levels were observed. Meanwhile, RT-PCR was used to investigate the mRNA expression of p53 in HCT116 cells. We observed that the mRNA expression levels of p53 significantly increased in a dose-dependent manner (Fig. 12C).

The IL6R/PI3K/AKT pathway leads to the activation of various downstream signaling substrates that are responsible for tumor cell proliferation, apoptosis, and migration. To investigate whether SKN inhibited the IL6R/PI3K/AKT pathway and its downstream signaling, we measured some essential protein expression levels involved in this pathway. As shown in Fig. 12B, 2.5 and $5.0 \mu \mathrm{mol} / \mathrm{L}$ SKN obviously inhibited the protein expression of IL6R, PI3K, p-AKT, and p-MDM2. These results suggested that SKN exerted its antitumor function by directly blocking the IL6-mediated downstream signaling cascade and further promoting the mRNA and protein expression of $\mathrm{p} 53$.

\section{Discussion}

SKN (8-dihydroxy-2-[(1R)-1-hydroxy-4-methylpent-3-enyl] naphthalene-1,4-dione) is a naphthoquinone pigment with serious fat-soluble properties and the ability to transfer electrons [25]. The molecular formula of SKN is shown in Fig. 1A. Recent studies have demonstrated that SKN displays antitumor potential for CRC by inhibiting malignant cell proliferation and migration and inducing cancer cell apoptosis [26]. It has also been reported that a number of molecular targets are affected by SKN, such as NF-KB, DNA topoisomerase, $\beta$-catenin, tyrosine phosphatases, mitogen-activated protein kinase (MAPK), phosphatase and tensin homolog deleted on chromosome 10 (PTEN), steroid sulfatase, p53, tyrosine kinase, and proteasome. Despite its undoubted anticancer efficacy, the underlying molecular mechanism of SKN is still elusive [27]. Thus, our study has contributed to elucidating the mechanisms underlying SKN's effects on inhibiting the growth of tumors by prediction with network pharmacology and verification with cell experiments.

In the first part, an integrated system pharmacology approach was successfully applied to clarify the antitumor mechanism of SKN. We adopted network pharmacology approaches to identify potential antitumor genes, and 30 corresponding targets for CRC were selected. Then, through a network pharmacology strategy including a PPI network, GO enrichment analysis, and 10 KEGG signaling 
pathways related to the treatment of CRC, we predicted the potential targets of SKN. With subsequent studies systematically analyzing the C-T-P network, we found that the pathological mechanism of SKN in CRC might be related to IL6, AKT1, TP53, CASP3, CCND1, PI3K-Akt signaling pathway, Jak-STAT signaling pathway, colorectal cancer, apoptosis, FoxO signaling pathway, etc. According to the prediction results of network pharmacology and literature review, the top hub genes are highly expressed in CRC cells. Molecular docking verification suggested that SKN can treat CRC by inhibiting interleukin 6 receptors, thereby inhibiting PI3K-AKT signaling pathways. This network pharmacology strategy is a rapid method to predict drug targets for complex diseases. Because these targets and signaling pathways are predicted by a network pharmacology strategy, we will support our findings by conducting more experiments in future work.

In the second part, we verified the effects of SKN on stable cytotoxicity, suppression of proliferation, induction of apoptosis, and inhibition of migration in HCT116 cells. First, the results showed that SKN could significantly suppress the proliferation of HCT116 cells by detecting the expression of Ki67, and HCT116 cells exposed to SKN displayed marked accumulation in the S/G2 phase in a dose-dependent manner. Moreover, the results of Hoechst 33342 staining showed that SKN could significantly increase the dense and fragmented nuclei in HCT116 cells, and the apoptotic and necrotic cells were more obvious compared with control group by staining with Annexin V-FITC and propidium iodide. Finally, we found that SKN significantly inhibited the migration properties of HCT116 cells by wound healing and Transwell assays, and the inhibitory effect of SKN was proportional to the increasing concentration. To date, we have proven the effectiveness of SKN on CRC HCT116 cells; however, the specific mechanism is still unknown. Thus, we further explored the mechanism of SKN-induced inhibition of HCT116 cells according to the results of network pharmacology.

The tumor suppressor protein p53 appears to have inhibitory effects on the cellular processes of tumor cells. In vitro studies have shown that the increasing expression of p53 can trigger the mitochondrial pathway of apoptosis in tumor cells, which induces cell apoptosis by promoting the expression of caspase-3 [28]. Moreover, some investigators have shown that p53 can suppress the proliferation and migration of tumor cells, which is mediated by inhibiting the expression of CDK2 and MMP9 [29]. The $\mathrm{PI} 3 \mathrm{~K} / \mathrm{AKT}$ signaling pathway plays essential roles in regulating various key factors related to cell growth, survival, and proliferation in tumor cells and can be activated by an array of stimuli, including growth factors, cytokines, and hormones, through various membrane receptors, such as cytokine receptors that include interleukin 6 receptors (IL6Rs) [30]. The PI3K/AKT signaling pathway has also been reported to inhibit the expression of p53 by promoting the phosphorylation of MDM2 [31]. Recent Western blotting data showed that SKN could significantly increase the protein expression of p53 and Caspase-3 and decrease the protein expression of MMP-9 and CDK2. Meanwhile, the expression of IL6R, PI3K, p-AKT, and P-MDM2 in the SKN group was significantly lower than that in the control group. Thus, these results might indicate that SKN suppresses proliferation and migration and induces apoptosis by inhibiting the IL6R/PI3K/AKT signaling pathway in HCT116 cells. 


\section{Conclusion}

In summary, the results demonstrate that SKN can inhibit the proliferation and migration and promote the apoptosis of CRC cells. We combined network pharmacology-based prediction, molecular docking, and in vitro experiments to verify the targets of SKN and the anti-CRC mechanisms. We demonstrated that SKN inhibited the activity of the IL6R/PI3K/AKT pathway by blocking IL6 and promoted the mRNA and protein expression of $\mathrm{p} 53$, thereby affecting the key signaling proteins of proliferation, apoptosis, and migration in CRC cells (Fig. 12D). This research not only provides fresh insights for a deeper understanding of the molecular mechanism of SKN in the treatment of CRC but also offers an efficient method to accelerate the development and clinical utilization of natural products as antitumor drugs.

\section{Abbreviations}

SKN: Shikonin; C-T-P: Compound-Target-Pathway; CRC: Colorectal cancer: RT-PCR: Real-time PCR; PPI: protein-protein interaction; WB: Western blot; GO: Gene Ontology; TCM: Traditional Chinese medicine; KEGG: Kyoto Encyclopedia of Genes and Genomes; C-T: Components-Targets; BP: biological process; CC: cellular components; PBS: phosphate buffered saline; PI: propidium iodide; FITC: fluorescein 5isothiocyanate.

\section{Declarations}

\section{Acknowledgements}

Thanks for West China school of pharmacy Sichuan University providing Schrodinger software and technical support.

\section{Authors' contributions}

Peng Song and Zefeng Wang were responsible for conception and design of the research; Qianfei Cui and Xiaojing Lu investigated the data. Zefeng Wang and Yongjia Shi performed data cleaning and analyzed the data. Ling Shi and Wenjing Guo were responsible for figure drawing and table design. Peng Song and Zhiming Zhang reviewed and edited the manuscript. All authors have read and approved the final manuscript.

\section{Funding}

This work was supported by the National Natural Science Foundation of China (81803779) ; Gansu Province Science Foundation for Distinguished Young Scholars (20JR10RA348) and Gansu Province Science Foundation for B Program (1508RJZA018) from Gansu Provincial Sci. \& Tech. Department; the Open Project of Research Center of Traditional Chinese Medicine, Gansu Province ( zyzx-2020-zx1); the Open Project of Key Laboratory of Prevention and Treatment for Chronic Diseases by TCM in Gansu Province (GSMBKY2015-01); Gansu Province Health Industry Scientific Research Program Management 
Project (GWGL2014-53). Thanks for West China school of pharmacy Sichuan University providing Schrodinger software and technical support.

\section{Availability of data and materials}

The datasets used during this study are available from the corresponding author upon reasonable request.

\section{Ethics approval and consent to participate}

Not applicable.

\section{Consent to publication}

All authors have read and agreed to the published version of the manuscript.

\section{Competing interests}

The authors declared that this work was conducted in the absence of any commercial or financial relationship that could be construed as a potential confict of interest.

\section{Author details}

${ }^{1}$ The Research Center of Chiral Drugs, Innovation Research Institute of Traditional Chinese Medicine (IRI), Shanghai University of Traditional Chinese Medicine, Shanghai 201203, China. ${ }^{2}$ Honghe University, Mengzi 661199, China. ${ }^{3}$ Research Center of Traditional Chinese Medicine, Gansu Province, Gansu University of Chinese Medicine, Lanzhou 730000, China. ${ }^{4}$ Affiliated Hospital of Gansu University of Chinese Medicine, Lanzhou 730000, China. ${ }^{5}$ Key Laboratory of Prevention and Treatment for Chronic Diseases by TCM, Gansu Province, Lanzhou 730000, China.

\section{References}

1. Ren J, Feng J, Song W, et al. Development and validation of a metabolic gene signature for predicting overall survival in patients with colon cancer. Clin Exp Med. 2020; 20:535-544

2. Jeong SY, Chessin DB, Schrag D, et al. Re: Colon cancer survival rates with the new American Joint Committee on Cancer sixth edition staging. J Natl Cancer Inst. 2005; 97:1705-1706, 1706-1707

3. Wang S, Gao J, Li Q, et al. Study on the regulatory mechanism and experimental verification of icariin for the treatment of ovarian cancer based on network pharmacology. J Ethnopharmacol. 2020; 262:113189

4. Yang LN, Wu ZL, Yang ZJ, Li SG, Ouyang CS. Exploring Mechanism of Key Chinese Herbal Medicine on Breast Cancer by Data Mining and Network Pharmacology Methods. Chin J Integr Med. 2020 
5. Liu C, He L, Wang J, et al. Anti-angiogenic effect of Shikonin in rheumatoid arthritis by downregulating PI3K/AKT and MAPKs signaling pathways. J Ethnopharmacol. 2020; 260:113039

6. Guo C, He J, Song X, et al. Pharmacological properties and derivatives of shikonin-A review in recent years. Pharmacol Res. 2019; 149:104463

7. Chandimali N, Sun HN, Kong LZ, et al. Shikonin-induced Apoptosis of Colon Cancer Cells Is Reduced by Peroxiredoxin V Expression. Anticancer Res. 2019; 39:6115-6123

8. Liu TH, Tu WQ, Tao WC, et al. Verification of Resveratrol Inhibits Intestinal Aging by Downregulating ATF4/Chop/Bcl-2/Bax Signaling Pathway: Based on Network Pharmacology and Animal Experiment. Front Pharmacol. 2020; 11:1064

9. Qin T, Wu L, Hua Q, et al. Prediction of the mechanisms of action of Shenkang in chronic kidney disease: A network pharmacology study and experimental validation. J Ethnopharmacol. 2020; 246:112128

10. Xu HH, Li SM, Xu R, et al. Predication of the underlying mechanism of Bushenhuoxue formula acting on knee osteoarthritis via network pharmacology-based analyses combined with experimental validation. J Ethnopharmacol. 2020; 263:113217

11. Shi L, Wu QG, Zhang JC, et al. Mechanism of Shuang-Huang-Lian Oral Liquid for Treatment of Mycoplasmal Pneumonia in Children on Network Pharmacology. Comb Chem High Throughput Screen. 2020; 23:955-971

12. Huang S, Zhang Z, Li W, et al. Network Pharmacology-Based Prediction and Verification of the Active Ingredients and Potential Targets of Zuojinwan for Treating Colorectal Cancer. Drug Des Devel Ther. 2020; $14: 2725-2740$

13. Zhuang Z, Wen J, Zhang L, et al. Can network pharmacology identify the anti-virus and antiinflammatory activities of Shuanghuanglian oral liquid used in Chinese medicine for respiratory tract infection. Eur J Integr Med. 2020; 37:101139

14. Liang XZ, Li R, Xu B, et al. Systematic evaluation of the mechanisms of zoledronic acid based on network pharmacology. Comput Biol Chem. 2019; 83:107097

15. Zeng Q, Li L, Siu W, et al. A combined molecular biology and network pharmacology approach to investigate the multi-target mechanisms of Chaihu Shugan San on Alzheimer's disease. Biomed Pharmacother. 2019; 120:109370

16. Song Y, Wang H, Pan Y, Liu T. Investigating the Multi-Target Pharmacological Mechanism of Hedyotis diffusa Willd Acting on Prostate Cancer: A Network Pharmacology Approach. Biomolecules. 2019;9

17. Yu G, Luo Z, Zhou Y, et al. Uncovering the pharmacological mechanism of Carthamus tinctorius L. on cardiovascular disease by a systems pharmacology approach. Biomed Pharmacother. 2019; 117:109094

18. Zhang Y, Li X, Guo C, Dong J, Liao L. Mechanisms of Spica Prunellae against thyroid-associated Ophthalmopathy based on network pharmacology and molecular docking. BMC Complement Med Ther. 2020; 20:229 
19. Song X, Zhang Y, Dai E, Du H, Wang L. Mechanism of action of celastrol against rheumatoid arthritis: A network pharmacology analysis. Int Immunopharmacol. 2019; 74:105725

20. Song $P$, Chen $P$, Wang $D$, et al. Realgar transforming solution displays anticancer potential against human hepatocellular carcinoma HepG2 cells by inducing ROS. Int J Oncol. 2017; 50:660-670

21. Gao K, Song YP, Du X, Chen H, Zhao LT. Exploring multiple mechanisms of Qingjie Fanggan prescription for prevention and treatment of influenza based on systems pharmacology. Comput Biol Chem. 2020; 88:107307

22. Wang ZF, Hu YQ, Wu QG, Zhang R. Virtual Screening of Potential Anti-fatigue Mechanism of Polygonati Rhizoma Based on Network Pharmacology. Comb Chem High Throughput Screen. 2019; 22:612-624

23. Tantawy MA, El-Sherbeeny NA, Helmi N, et al. Synthetic antiprotozoal thiazolide drug induced apoptosis in colorectal cancer cells: implications of IL-6/JAK2/STAT3 and p53/caspases-dependent signaling pathways based on molecular docking and in vitro study. Mol Cell Biochem. 2020; 469:143157

24. Qiu CZ, Wang C, Huang ZX, et al. Relationship between somatostatin receptor subtype expression and clinicopathology, Ki-67, Bcl-2 and p53 in colorectal cancer. World J Gastroenterol. 2006; 12:20112015

25. Guo C, He J, Song X, et al. Pharmacological properties and derivatives of shikonin-A review in recent years. Pharmacol Res. 2019; 149:104463

26. Chandimali N, Sun HN, Kong LZ, et al. Shikonin-induced Apoptosis of Colon Cancer Cells Is Reduced by Peroxiredoxin V Expression. Anticancer Res. 2019; 39:6115-6123

27. Zhang X, Cui JH, Meng QQ, et al. Advance in Anti-tumor Mechanisms of Shikonin, Alkannin and their Derivatives. Mini Rev Med Chem. 2018; 18:164-172

28. Neves SP, de Carvalho NC, Da SM, et al. Ruthenium Complexes Containing Heterocyclic Thioamidates Trigger Caspase-Mediated Apoptosis Through MAPK Signaling in Human Hepatocellular Carcinoma Cells. Front Oncol. 2019; 9:562

29. Song P, Chen P, Wang D, et al. Realgar transforming solution displays anticancer potential against human hepatocellular carcinoma HepG2 cells by inducing ROS. Int J Oncol. 2017; 50:660-670

30. Slattery ML, Mullany LE, Sakoda LC, et al. The PI3K/AKT signaling pathway: Associations of miRNAs with dysregulated gene expression in colorectal cancer. Mol Carcinog. 2018; 57:243-261

31. Liu L, Yan J, Cao Y, et al. Proliferation, migration and invasion of triple negative breast cancer cells are suppressed by berbamine via the PI3K/Akt/MDM2/p53 and PI3K/Akt/mTOR signaling pathways. Oncol Lett. 2021; 21:70

\section{Tables}

Table1. The core targets information table 


\begin{tabular}{|llll|}
\hline Targets & Degree & Targets & Degree \\
\hline TP53 & 12 & IL10 & 9 \\
\hline CCND1 & 11 & KDR & 8 \\
\hline CASP3 & 10 & PPARG & 8 \\
\hline AKT1 & 10 & BCL2 & 4 \\
\hline IL4 & 9 & PCNA & 2 \\
\hline CXCL8 & 9 & EPHB2 & 1 \\
\hline IL6 & 9 & & \\
\hline
\end{tabular}

Table2. Compound-target-pathway network 


\begin{tabular}{|c|c|c|c|c|}
\hline Classification & Term & Degree & Betweenness & Closeness \\
\hline Compound & SKN & 11 & 0.30749554 & 0.67741935 \\
\hline Pathway 1 & PI3K-Akt signaling pathway & 8 & 0.10977198 & 0.56756757 \\
\hline Pathway 2 & Jak-STAT signaling pathway & 5 & 0.03903222 & 0.48837209 \\
\hline Pathway 3 & Colorectal cancer & 5 & 0.02116617 & 0.44680851 \\
\hline Pathway 4 & Apoptosis & 4 & 0.01189025 & 0.42857143 \\
\hline Pathway 5 & FoxO signaling pathway & 4 & 0.02137697 & 0.46666667 \\
\hline Pathway 6 & Transcriptional misregulation in cancer & 4 & 0.02982116 & 0.46666667 \\
\hline Pathway 7 & Focal adhesion & 4 & 0.022081 & 0.46666667 \\
\hline Pathway 8 & Cytokine-cytokine receptor interaction & 4 & 0.01865581 & 0.41176471 \\
\hline Pathway9 & MicroRNAs in cancer & 4 & 0.01187533 & 0.42857143 \\
\hline Pathway 10 & $\begin{array}{l}\text { Intestinal immune network for IgA } \\
\text { production }\end{array}$ & 3 & 0.00683357 & 0.39622642 \\
\hline Target1 & IL6 & 8 & 0.15044895 & 0.56756757 \\
\hline Target 2 & AKT1 & 7 & 0.09128616 & 0.53846154 \\
\hline Target 3 & CCND1 & 6 & 0.06730637 & 0.51219512 \\
\hline Target 4 & CASP3 & 6 & 0.05777487 & 0.51219512 \\
\hline Target 5 & TP53 & 6 & 0.06821235 & 0.51219512 \\
\hline Target 6 & BCL2 & 5 & 0.03433465 & 0.48837209 \\
\hline Target 7 & CXCL8 & 5 & 0.0478964 & 0.48837209 \\
\hline Target 8 & IL10 & 5 & 0.04588149 & 0.48837209 \\
\hline Target 9 & IL4 & 4 & 0.03054306 & 0.466666667 \\
\hline Target 10 & PCNA & 2 & 0.00164839 & 0.42857143 \\
\hline Target 11 & PPARG & 2 & 0.0046673 & 0.42857143 \\
\hline
\end{tabular}

\section{Figures}


A<smiles>CC(C)=CC[C@H](O)C1=CC(=O)c2c(O)ccc(O)c2C1=O</smiles>

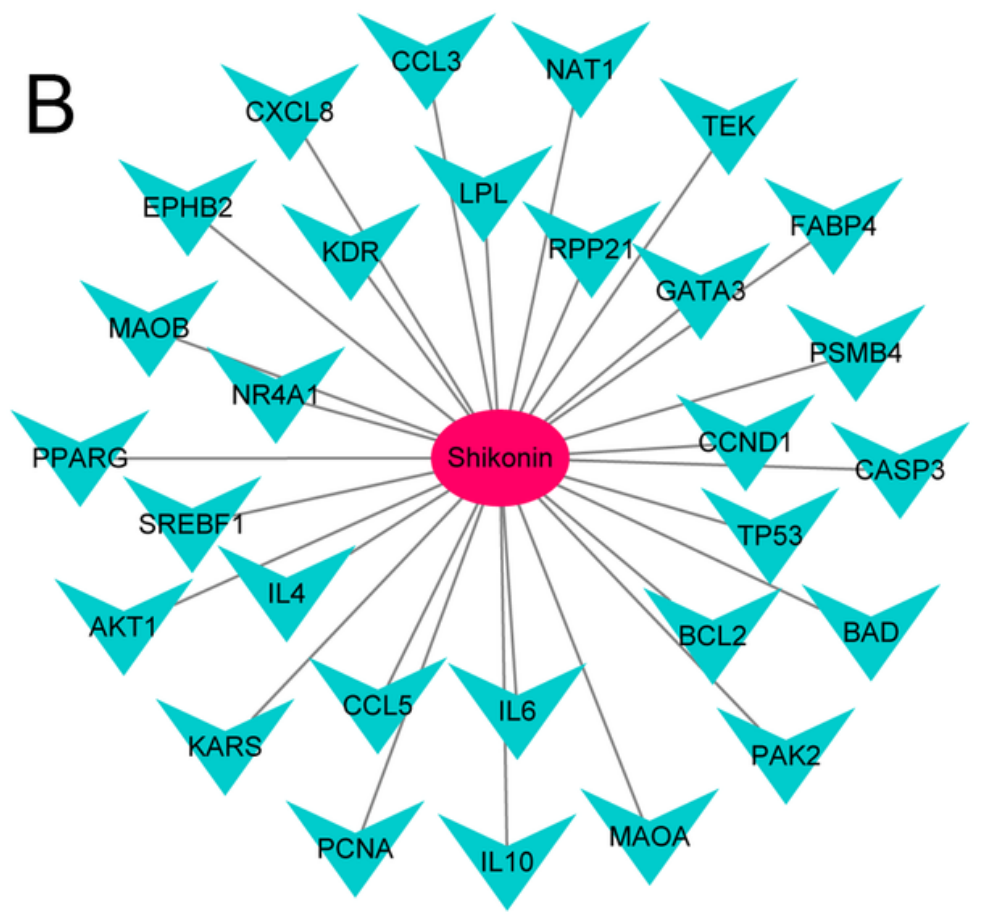

Figure 1

Compound-target network of SKN. The red ellipse represents the SKN and V-shape represents the targets of the SKN, the edges represent the interaction of them. 


\section{Shikonin}

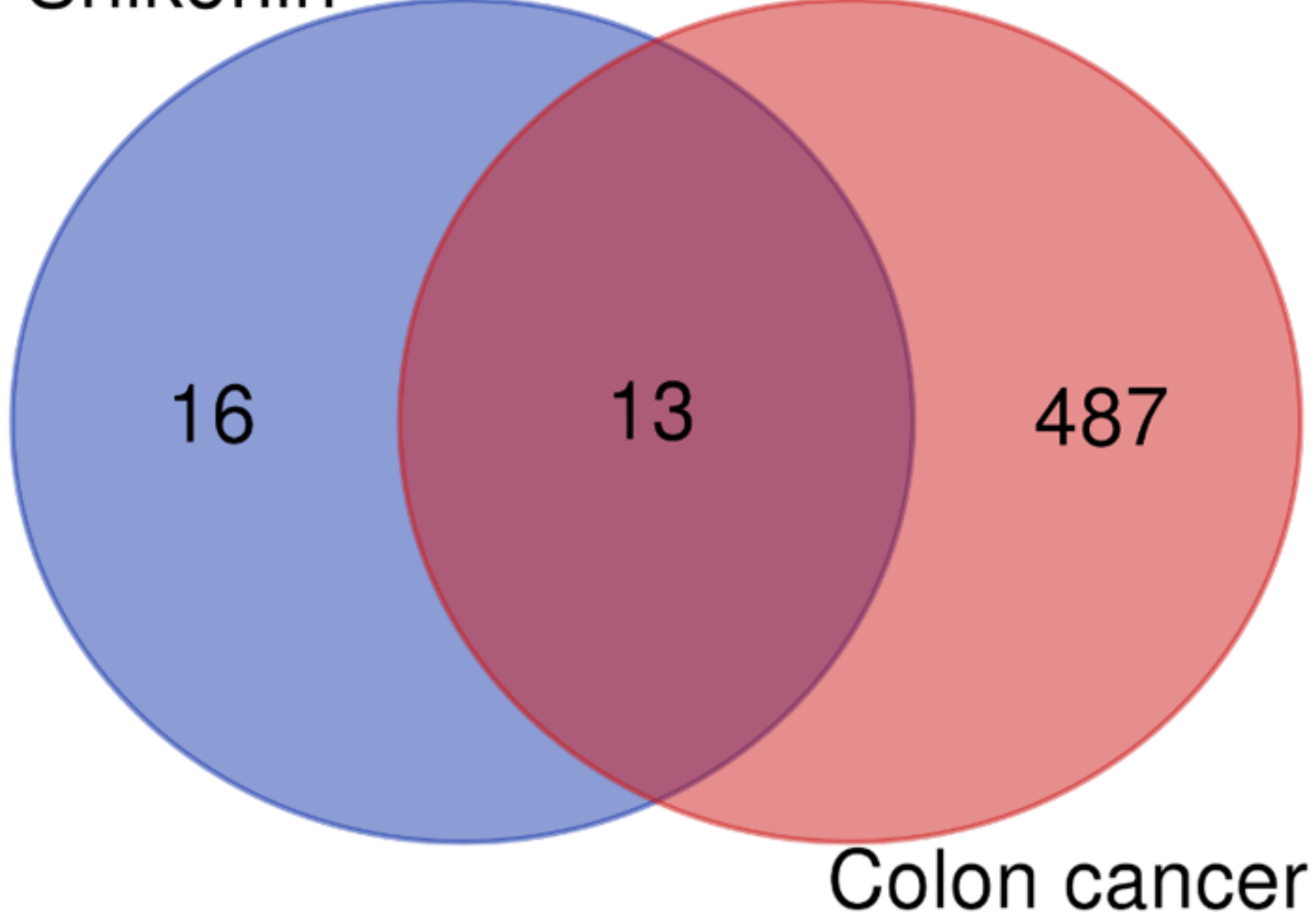

Figure 2

Venn diagram of intersection targets of SKN and CRC. 


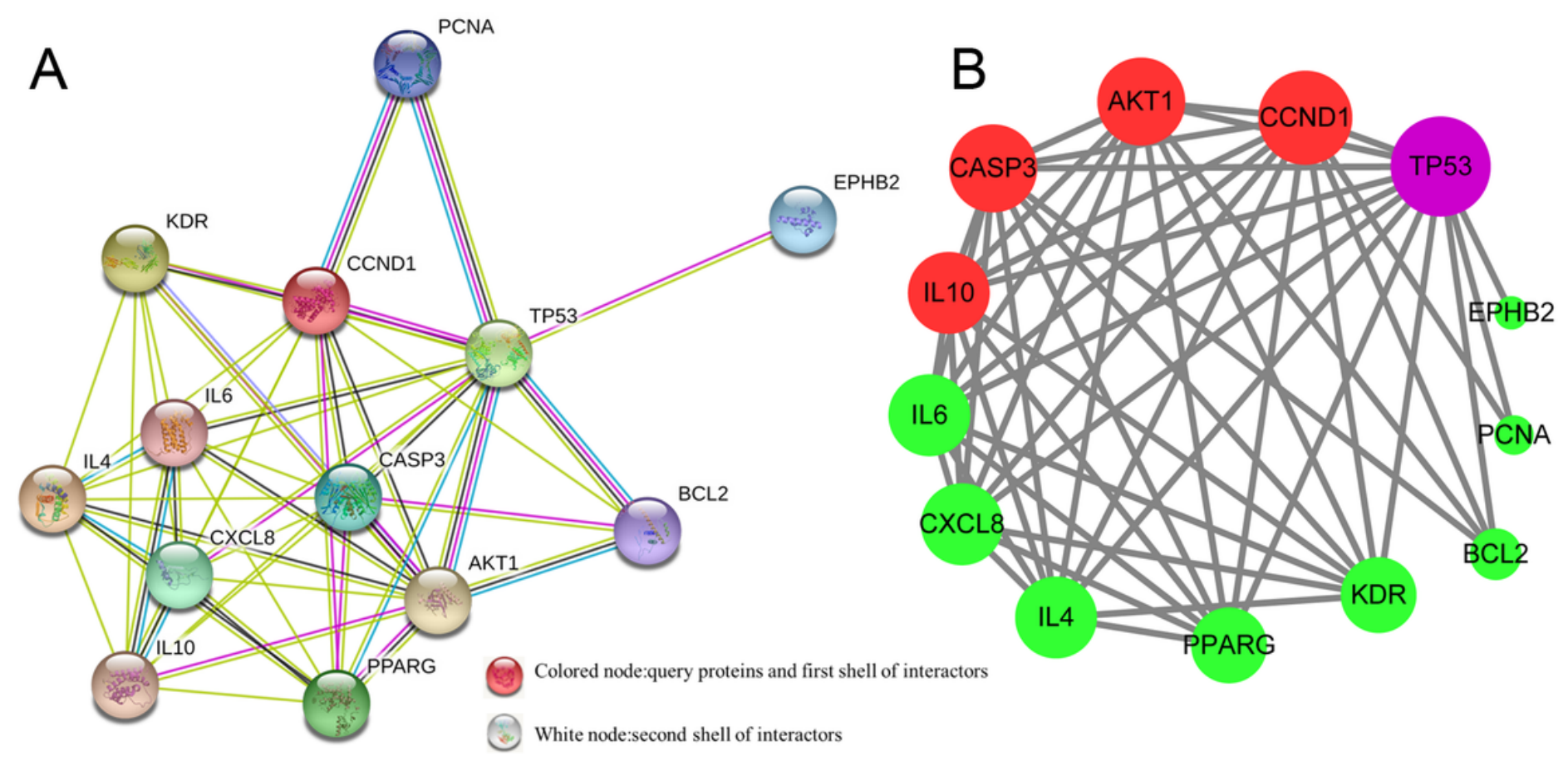

Figure 3

(A)Interaction network of target proteins related to SKN in the treatment of CRC. (B) The PPI network of SKN in the treatment of CRC. The edges represent the interaction of them. According to the degree value, from large to small. 


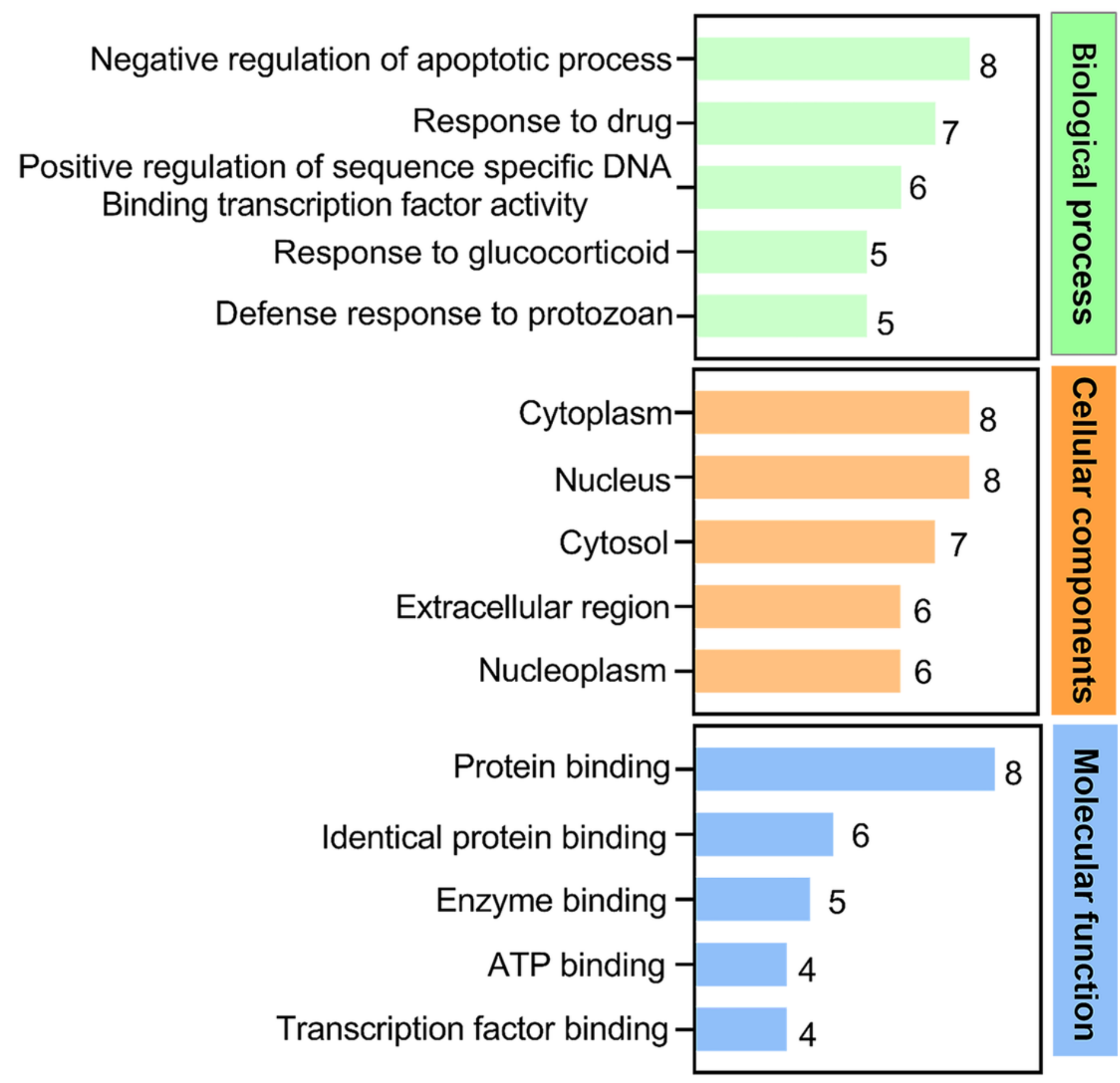

Number of proteins

Figure 4

The GO enrichment analysis of potential targets of SKN in the treatment of CRC. The top 5 terms of each part are shown: biological process (BP), cell component (CC), molecular unction (MF). 


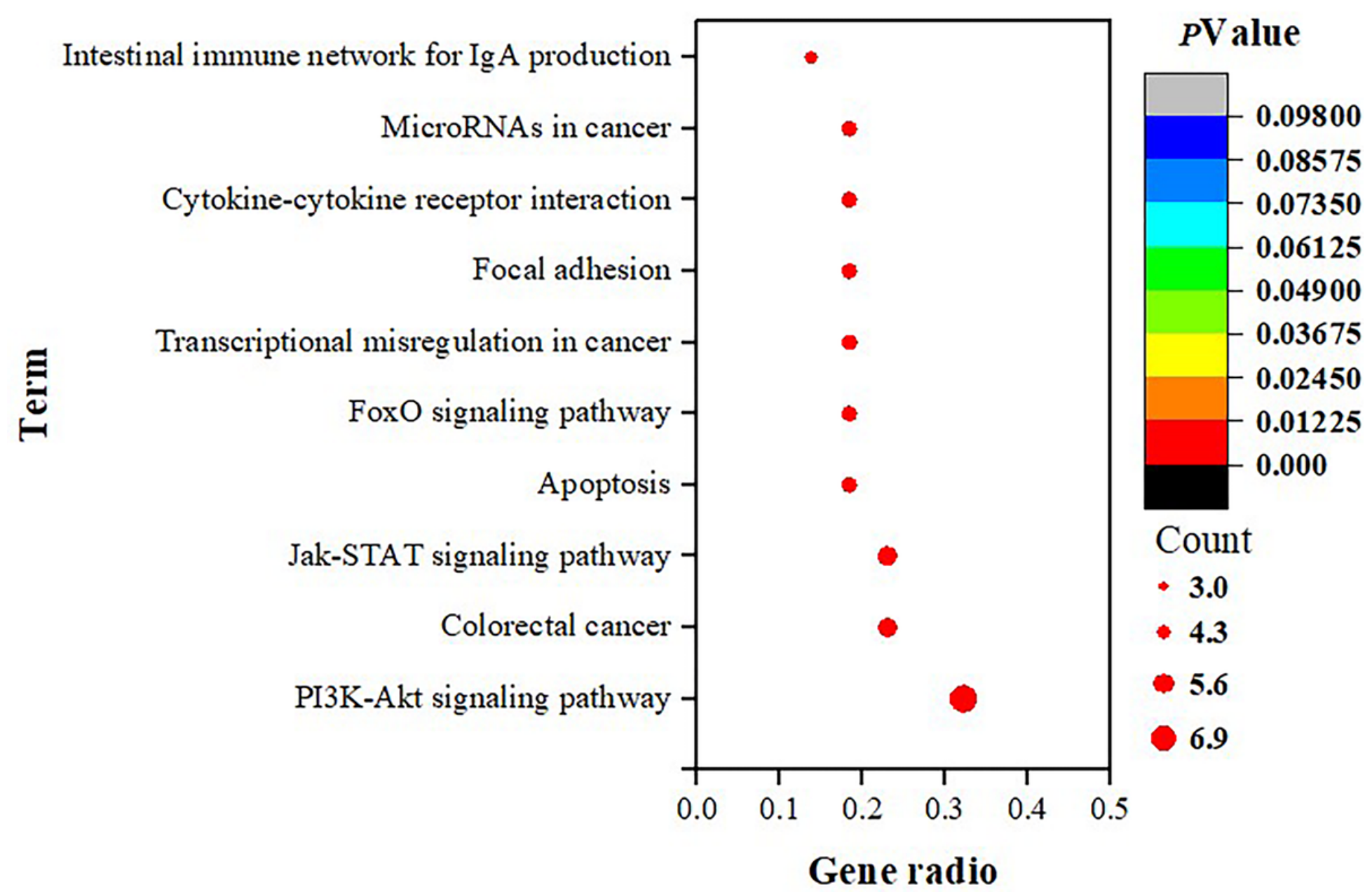

Figure 5

Bubble chart signaling pathways of KEGG pathway enriched by major hubs from cluster profiler. The $\mathrm{x}$ axis shows the gene ratio and the $y$-axis shows significantly enriched signal pathways of the target genes, Gene Ratio stands for the ratio of the number of target genes belonging to signal pathway to the number of all the annotated genes. The higher gene ratio represents the higher level of enrichment. The larger the dot size, the more the number of target genes in the pathway, and the deeper of the red color the dot is, the smaller of $p$ value. 


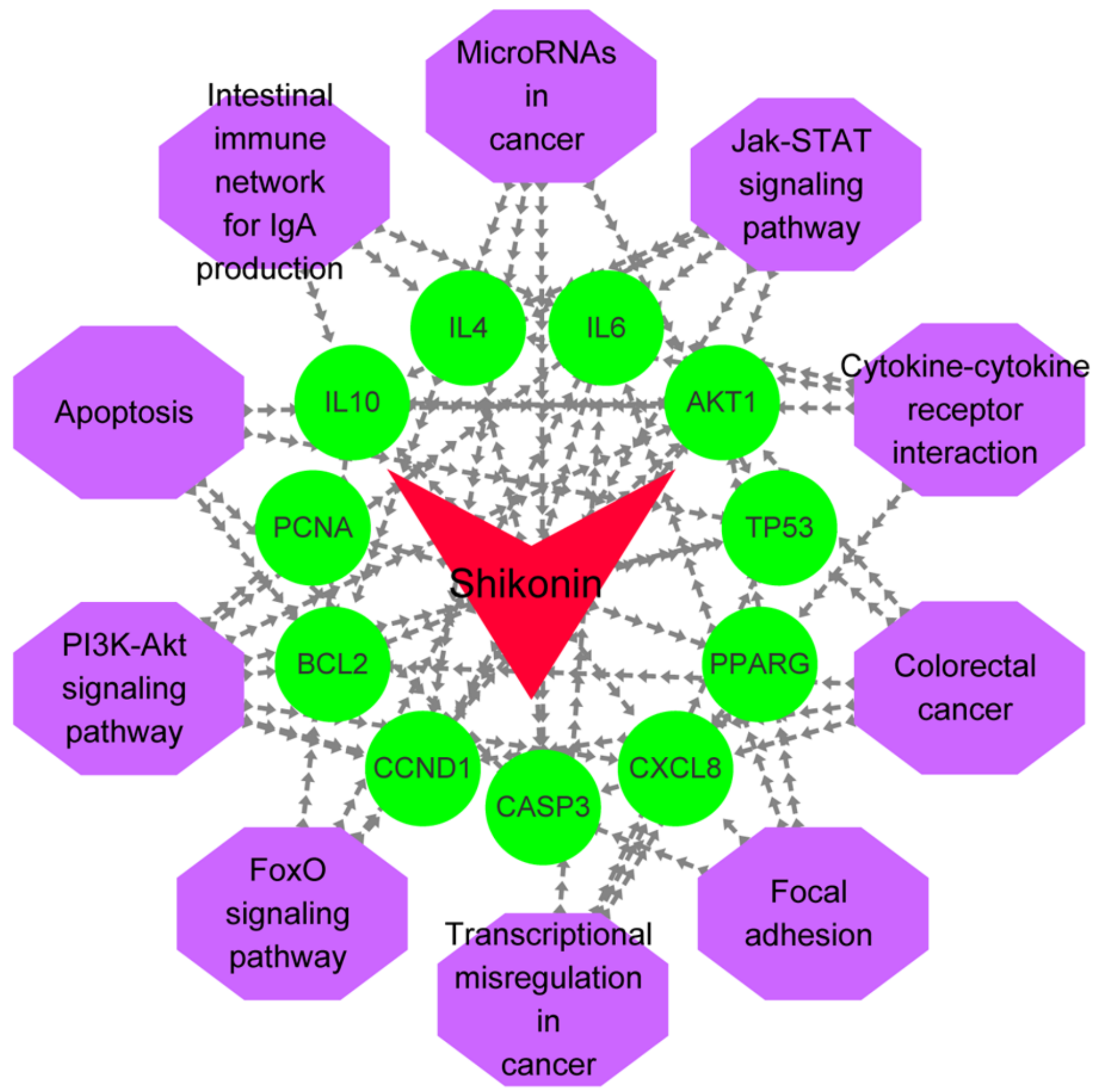

Figure 6

Compound-target-pathway network in the treatment of CRC. The red V represent the drug; the Green circle represent the target corresponding to SKN, and the purple polygon represent the pathways enriched with the targets, the edges represent the interaction of them. 


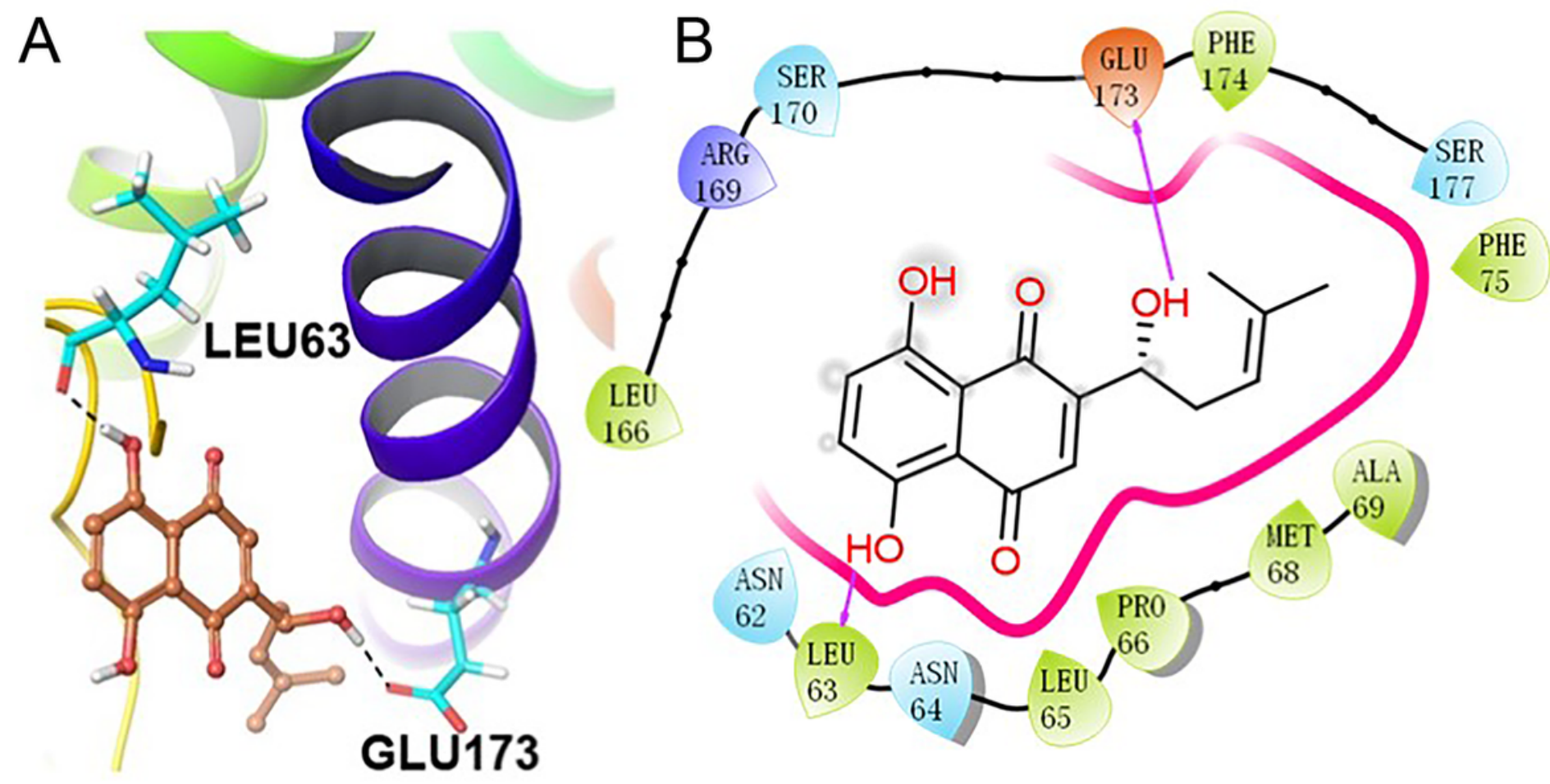

Figure 7

The docking complex of targets and component. A: Binding interaction of SKN in IL6. B: The 2D Ligplot of SKN against IL6 


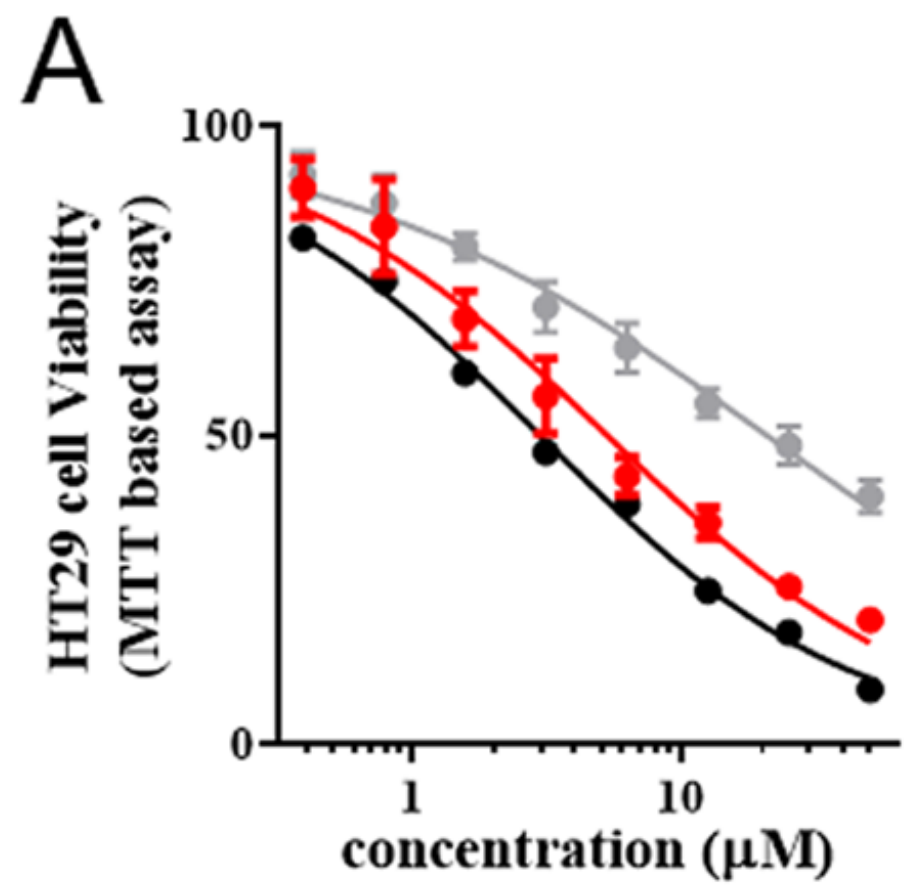

B
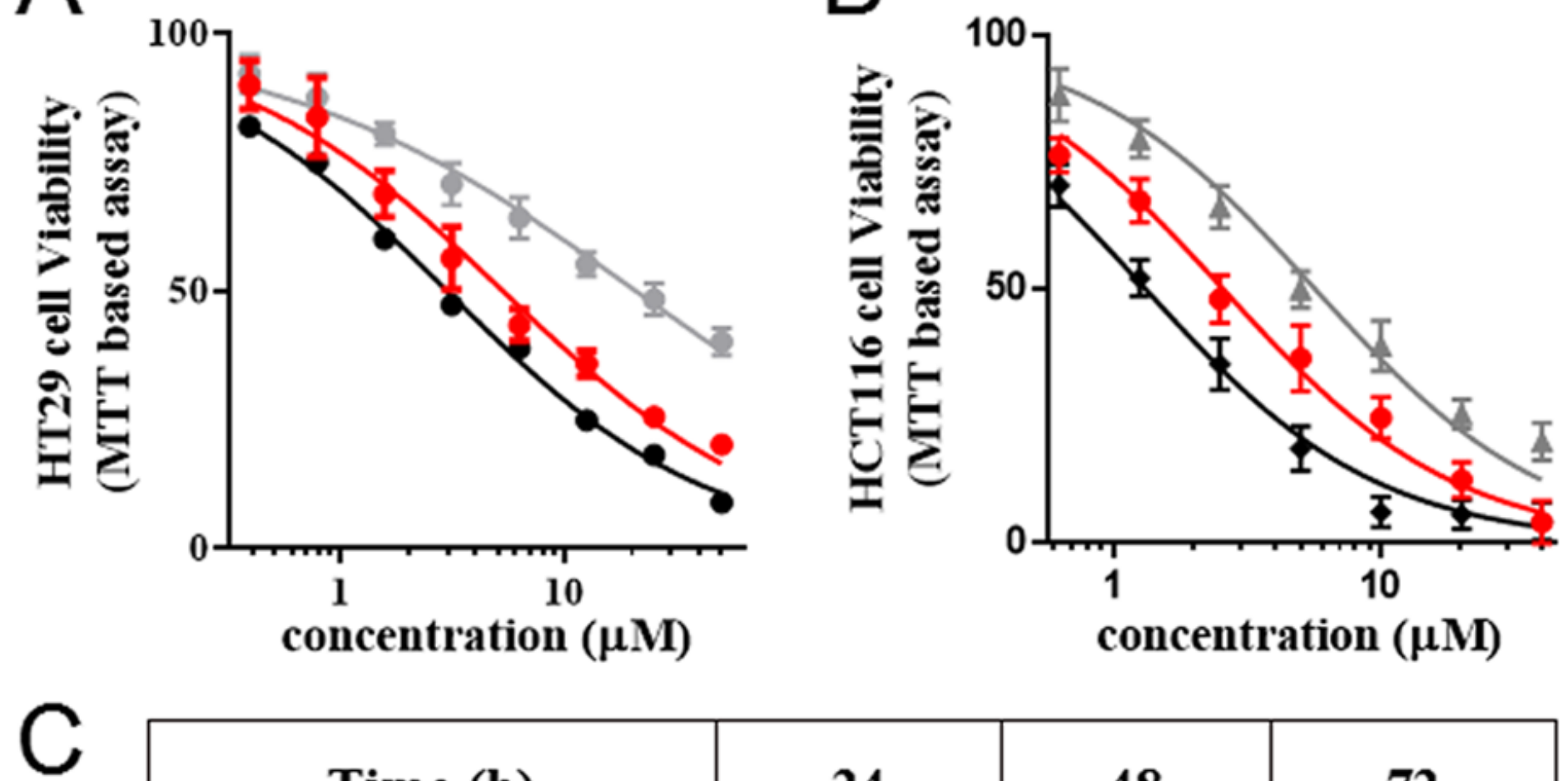

\begin{tabular}{|c|c|c|c|c|}
\hline \multicolumn{2}{|c|}{ Time (h) } & 24 & 48 & 72 \\
\hline \multirow{2}{*}{$\begin{array}{c}\text { IC50 } \\
(\mu M)\end{array}$} & HT29 & 21.75 & 5.565 & 2.96 \\
\cline { 2 - 5 } & HCT116 & 5.486 & 2.568 & 1.318 \\
\hline
\end{tabular}

Figure 8

A: Dose- and time-dependent cytotoxicity of SKN with the indicated concentrations to HT29 cells ( $n=8$ for each concentration in each group). B: Dose-dependent cytotoxicity of SKN with the indicated concentrations to HCT116 cells ( $n=8$ for each concentration in each group). C: The IC50 values for inhibiting HT29 and HCT116 cells on different times. 
Control
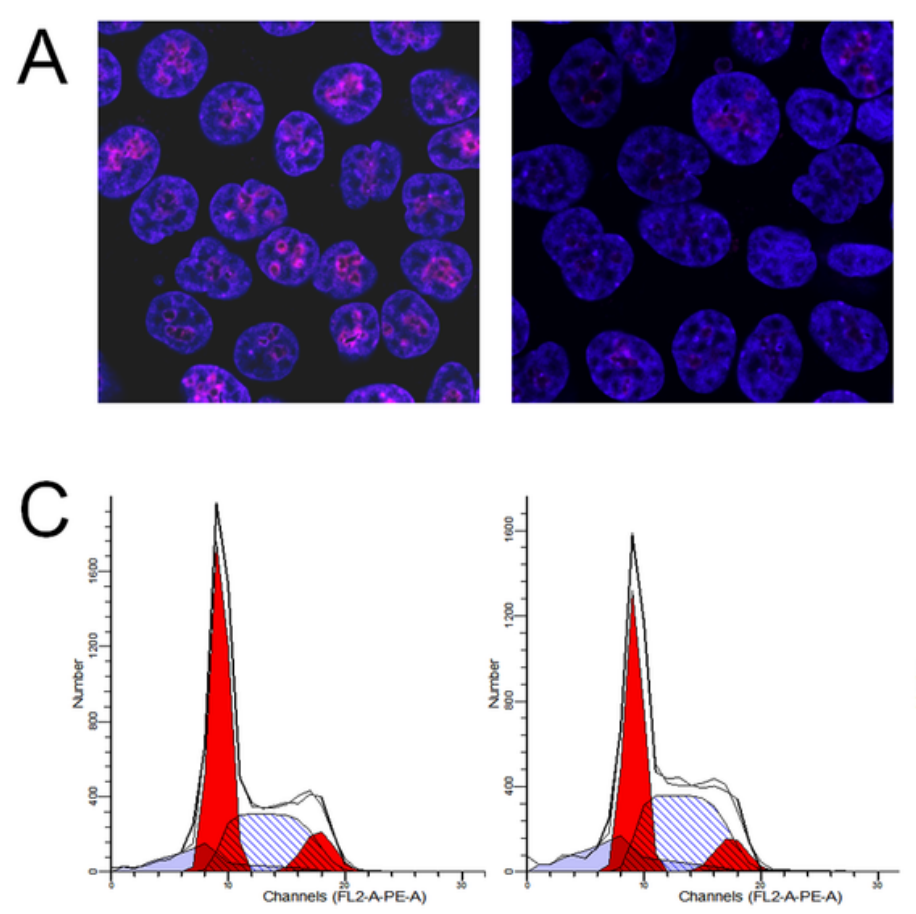

SKN 5
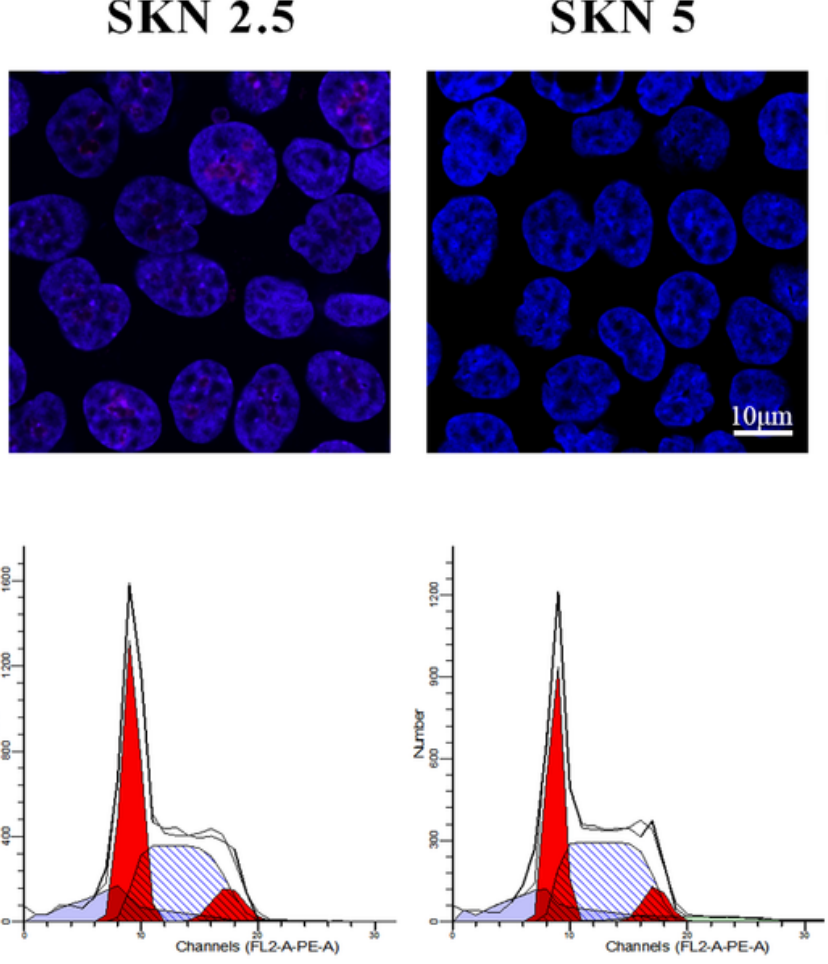

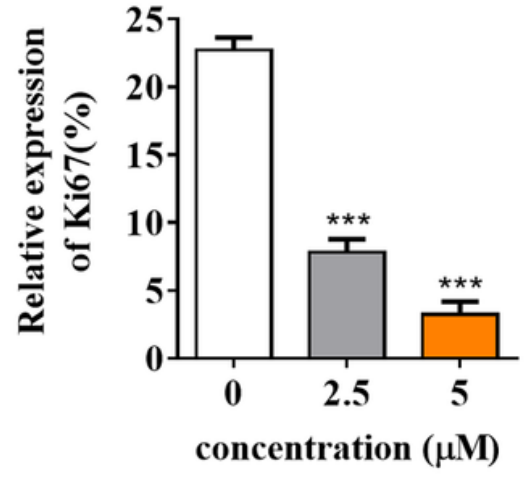

$\square \mathrm{G} 1 \quad \square \mathrm{S} \quad \square \mathrm{G} 2$

D

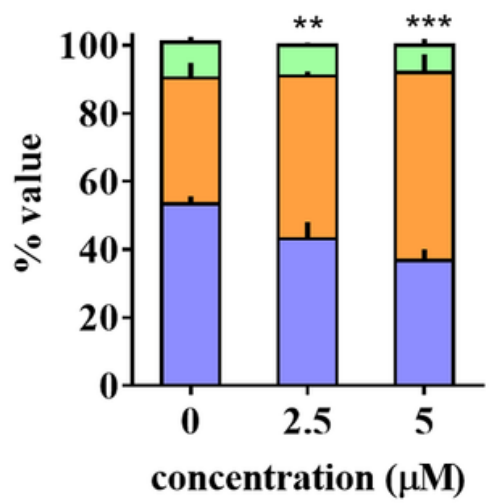

Figure 9

A: Representative images of Ki67 immunofluorescence in HCT116 cells treated with different concentrations of SKN $(\times 1000)$. Ki67 protein was marked by pink fluorescence, and nucleus was marked by blue fluorescence (DAPI staining). B: The protein expression of Ki67 was quantified by Image $\mathrm{J}$ software. C and D: Cell cycle was evaluated by flow cytometry after SKN treating for $48 \mathrm{~h}$, and the percentage of cell cycle distribution was presented in histograms (Results shown are representative of three independent experiments, ${ }^{\star *} P<0.01$ vs control, ${ }^{\star \star *} P<0.001$ vs control). 
Control
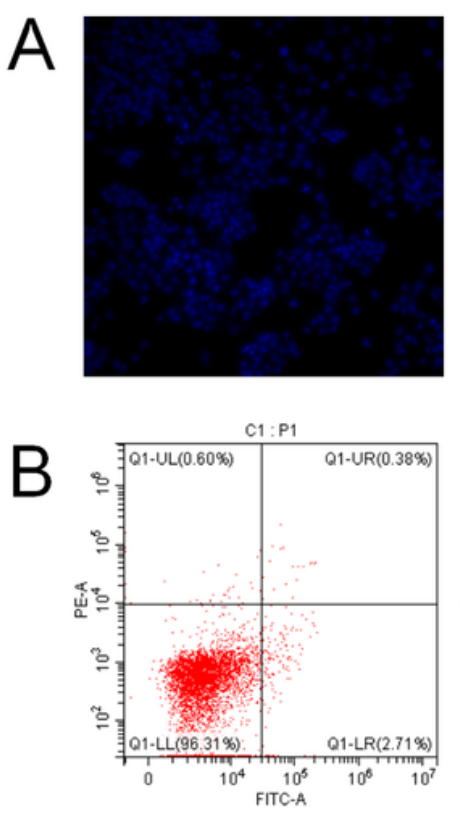

SKN 2.5
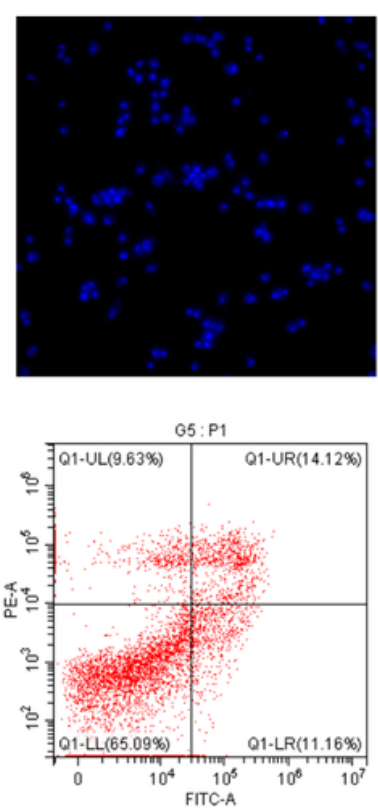

SKN 5
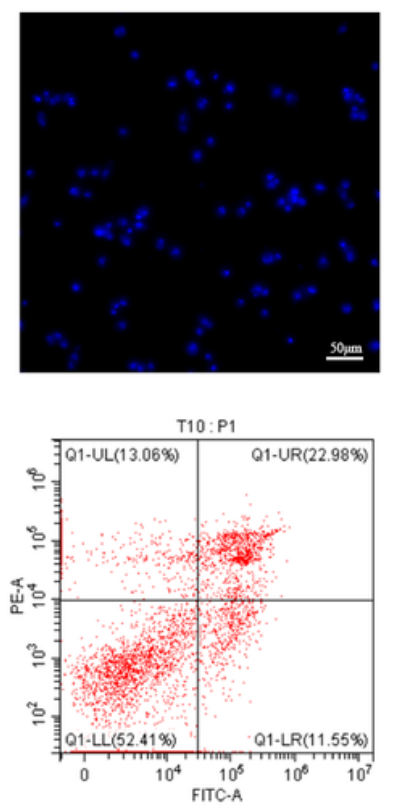

C

$\square$ normal $\square$ apoptosis $\square$ necrosis

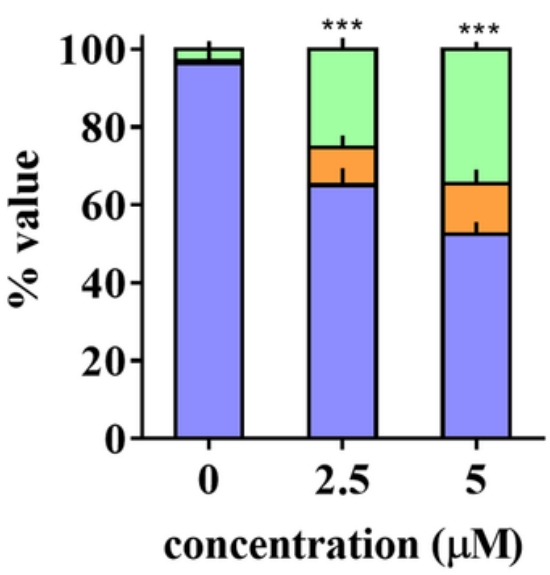

Figure 10

A: Representative images of nuclear condensation in HCT116 cells treated with different concentrations of SKN by Hoechst 33342 staining $(\times 100)$. B and C: Cell apoptosis was evaluated by annexin V/PI double-staining assay after SKN treating for $48 \mathrm{~h}$, and the percentage of cell distribution was presented in histograms (Results shown are representative of three independent experiments, $* \star P<0.01$ vs control, $* \star \star$ $\mathrm{P}<0.001$ vs control). 


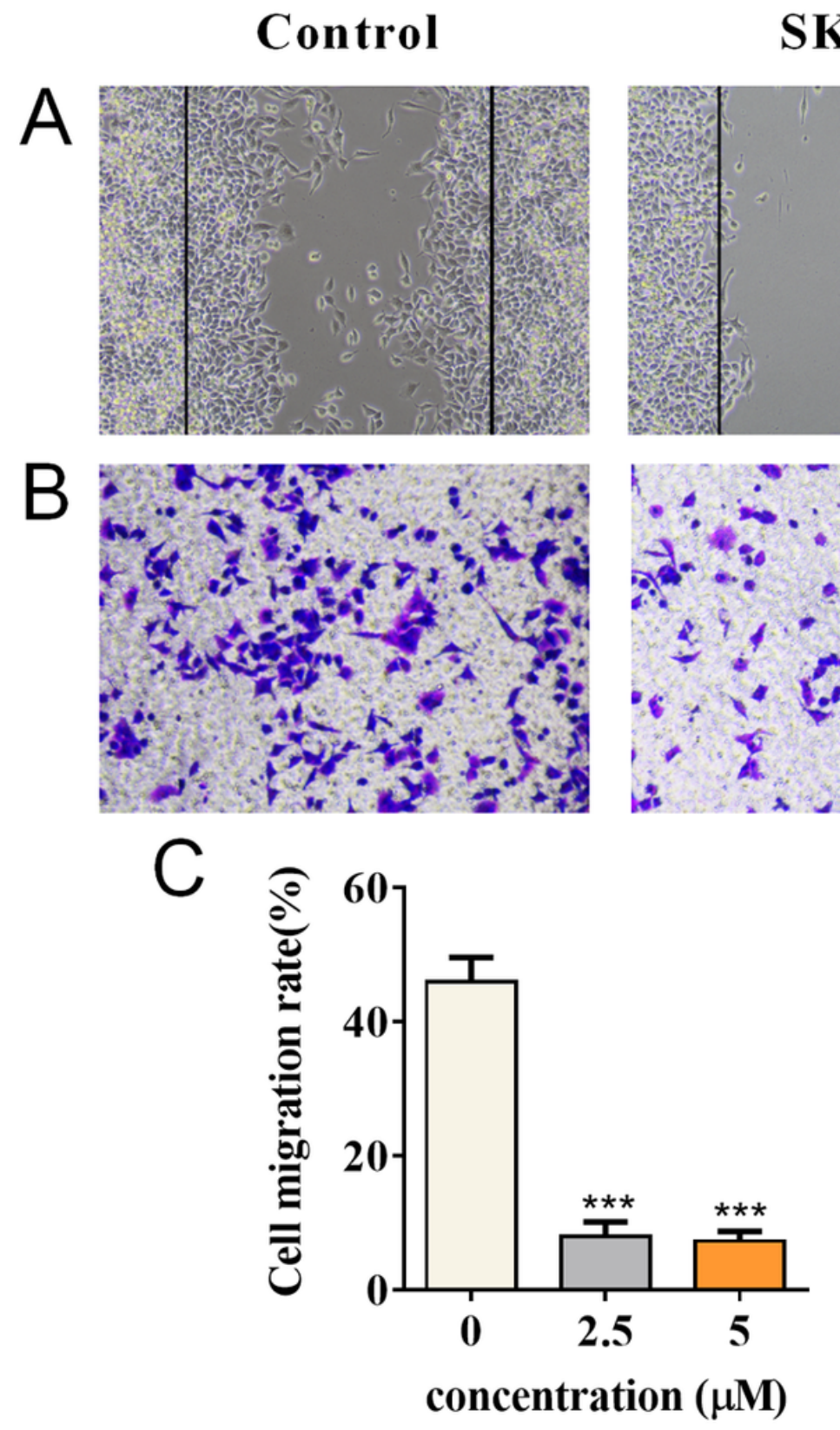

SKN 2.5

SKN 5
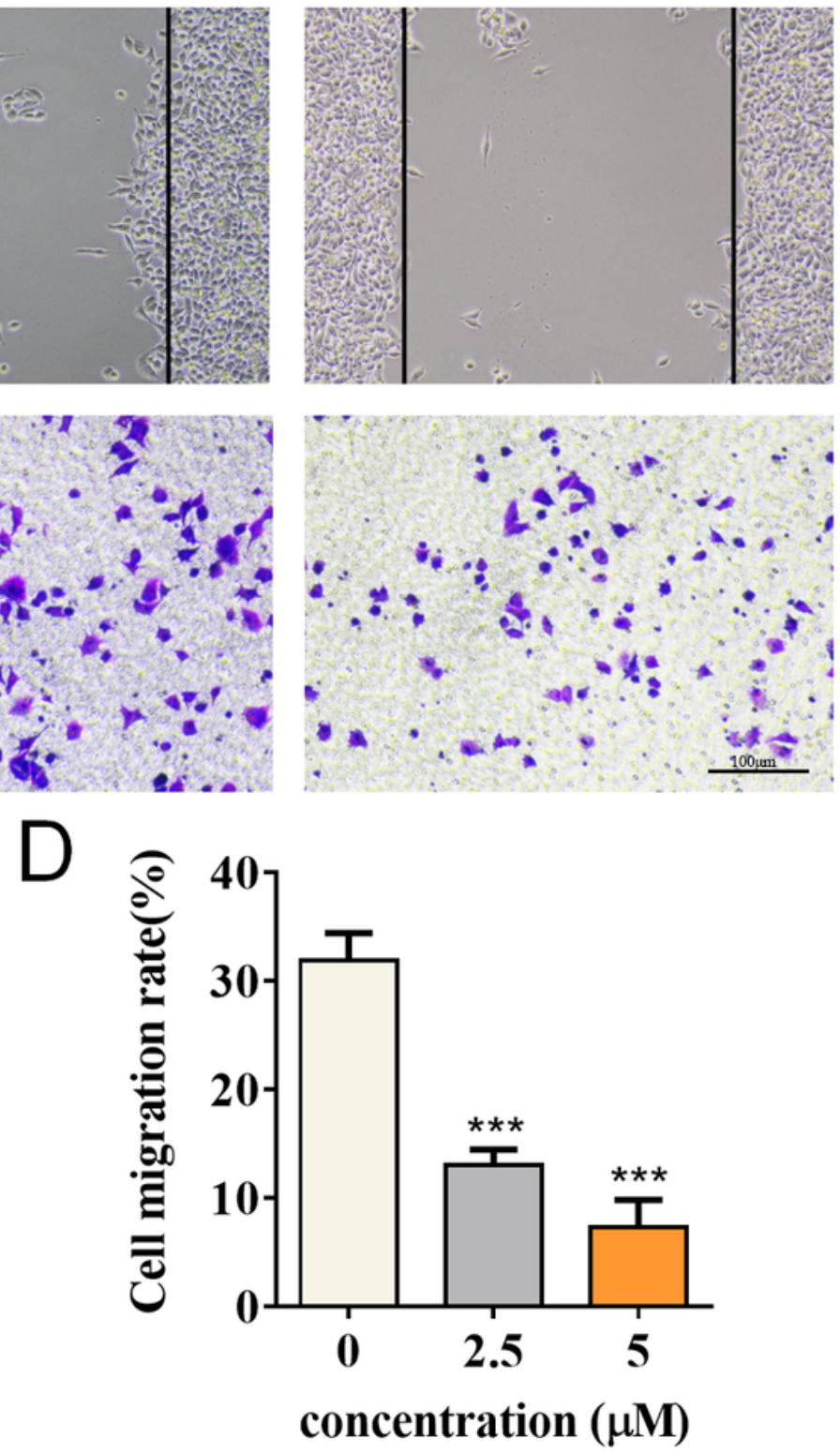

Figure 11

A and C: Representative images of HCT116 cells migration treated with different concentrations of SKN by wound healing assay $(\times 100)$, and the rate of cell migration was presented in histograms. $B$ and $D$ : Representative images of HCT116 cells migration treated with different concentrations of SKN by Transwell assay $(\times 200)$, and the rate of cell migration was presented in histograms (Results shown are representative of three independent experiments, $* * P<0.01$ vs control, $* * * P<0.001$ vs control). 


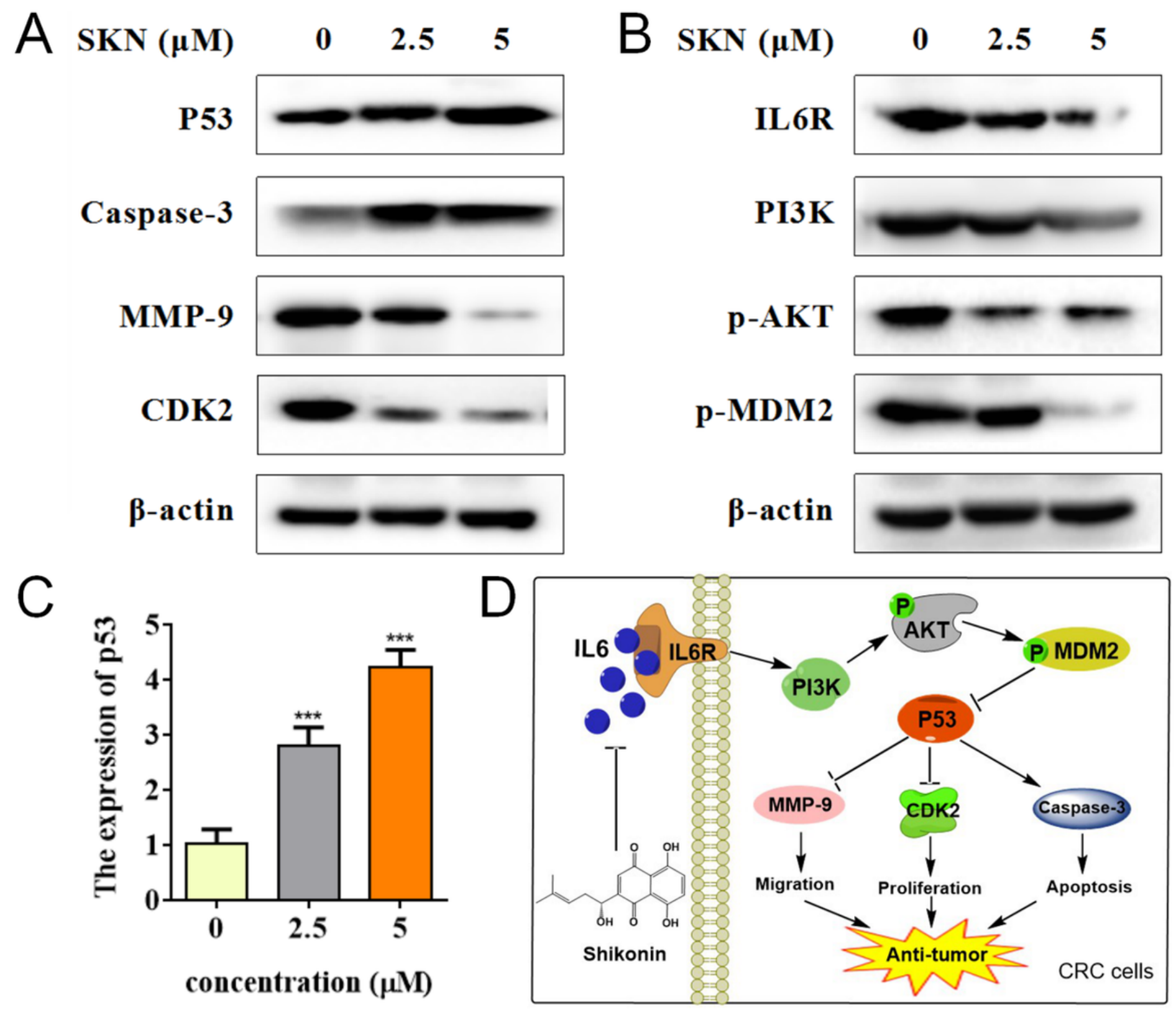

Figure 12

A: Representative images of Western blots showing the protein expression of p53 and its downstream Caspase-3, MMP-9, and CDK2 in HCT116 cells. B: Representative images of Western blots showing the protein expression of IL6R/PI3K/AKT pathway in HCT116 cells, and $\beta$-actin was used as a loading control. C: The mRNA level of p53 genes in HCT116 cells was analyzed by qRT-PCR, and the relative expression of $p 53$ was presented in histograms $(n=3, * \star P<0.01$ vs control, $* \star \star ~ P<0.001$ vs control). D: diagram of signaling pathways for SKN-regulated anti-tumor in CRC cells.

\section{Supplementary Files}

This is a list of supplementary files associated with this preprint. Click to download. 
- AbstractFingure.png

Page 30/30 